\title{
Pobreza transitoria, nuevos enfoques a partir de la crisis económica
}

\section{Transitional Poverty, New Approaches from the Economic Crisis}

María del Carmen Pérez-Peña (i) https://orcid.org/0000-0002-9242-4286

Universidad de Cádiz (UCA), España, carmen.perezpena@uca.es

Recepción:

$30 / 05 / 18$

Aprobación: 08/02/19

\author{
Mercedes Jiménez-García (i) https://orcid.org/0000-0002-4180-2216 \\ Universidad de Cádiz(UCA), España, mercedes.jimenezgarcia@uca.es \\ María del Carmen Pérez-González (D) https://orcid.org/0000-0002-3028-5268 \\ Universidad de Cádiz (UCA), España, maricarmen.perez@uca.es
}

\begin{abstract}
The current economic crisis is revealing social and structural changes that entail a worsening of the levels of poverty and wellbeing of the population. In this line, the objective of this work is to deepen the knowledge of the situation of poverty in the municipality of Jerez de la Frontera, to be able to contribute to a better design and application of measures and actions of general and specific socioeconomic policies with greater guarantee of success. For this, an analysis methodology has been applied by age, sex, productive sectors and other characteristics based on surveys and interviews. Among the main conclusions of this work and as one of its contributions, the emergence of a new type of transitory poverty from the construction sector should be noted, establishing a new scenario derived from the impact of the crisis, which requires a specific response.
\end{abstract}

Key words: construction sector poverty, economic crisis, chronic poverty, recurrent transient poverty and non-recurrent transient poverty.

Resumen: La actual crisis económica está poniendo de manifiesto cambios sociales y estructurales, que conllevan un empeoramiento de los niveles de pobreza y de bienestar de la población. En esta línea, el objetivo de este trabajo es profundizar en el conocimiento de la situación de pobreza en el municipio de Jerez de la Frontera, para contribuir a un mejor diseño y aplicación de medidas y actuaciones de políticas socioeconómicas generales y específicas con mayor garantía de éxito. Para ello, se ha aplicado una metodología de análisis por edad, sexo, sectores productivos y otras características, basada en encuestas y entrevistas. Entre las principales conclusiones de este trabajo y como una de sus contribuciones, hay que señalar la aparición de un nuevo tipo de pobreza transitoria, proveniente del sector de la construcción, estableciéndose un nuevo escenario derivado del impacto de la crisis, el cual requiere de una respuesta específica.

Palabras clave: pobreza del sector de la construcción, crisis económica, pobreza crónica, pobreza transitoria recurrente y pobreza transitoria no recurrente. 


\section{Introducción}

La actual crisis internacional iniciada en 2007 ha provocado modificaciones en los escenarios económicos, propiciando el descenso de la inversión, la destrucción del tejido empresarial con la consecuente caída del empleo, la reducción de la renta disponible, de la demanda y del consumo, y los recortes en el gasto público que han afectado directamente al bienestar social, incidiendo en la calidad y en las condiciones de vida de la población. Todo esto se manifiesta tanto en el notable incremento que han experimentado los actuales indicadores de pobreza, como en el cambio en su estructura y composición sectorial y territorial, que ralentizan el crecimiento económico y provoca aumentos considerables en la desigualdad (Rocha, 2012; Conde et al., 2016; Sánchez-López y Paz-Báñez, 2016).

En el caso español, este impacto de la crisis se ha ido agudizando hasta 2014, reflejándose en la evolución seguida por la tasa At Risk of Poverty and Exclusion (AROPE), ${ }^{1}$ pasando de un 23,8\% en 2008 a un 29,2\% en 2014. A partir de ahí, este indicador ha experimentado una ligera mejoría, $-27,9 \%$ en 2016 (EAPN, 2017), manteniéndose, no obstante, los cambios provocados por la crisis en la composición de la pobreza.

Además de los elevados niveles de los indicadores, la desigual distribución de su impacto en el territorio agrava la situación en comunidades autónomas tradicionalmente más desfavorecidas, como es el caso de Andalucía, y, dentro de ellas, en municipios particularmente sensibles por su no consolidada estructura económica y social, como puede ser el caso de Jerez de la Frontera, ${ }^{2}$ en la provincia de Cádiz.

El objetivo de este trabajo es profundizar en el conocimiento de la pobreza, en general, y de la pobreza transitoria, en concreto, a partir del planteamiento - como cuestión de investigación- de si puede considerarse la existencia de un cambio en la composición de la pobreza transitoria, definido por la presencia de un nuevo tipo de pobreza no identificado previamente y surgido a partir del impacto de la crisis, sobre todo, en el periodo de mayor intensidad y efecto en los indicadores (2008-2014).

Para desarrollar este trabajo de carácter empírico y exploratorio, se efectuó una serie de encuestas y entrevistas ante la falta de información oficial a nivel local, con el fin de responder a la cuestión de investigación planteada.

1 La tasa AROPE incluye la tasa de riesgo de pobreza, la baja intensidad en el empleo y la carencia material severa (Arnal, 2013; FOESSA, 2014; Malgesini y Candalija, 2014).

2 Los datos de desempleo en Jerez de la Frontera se situaban en $29.13 \%$ en 2008, 38.95\% en 2014 y $36.26 \%$ en 2016 (SIMA, 2017). 
El propósito es poner de manifiesto la existencia de un nuevo tipo de pobreza en el municipio de Jerez de la Frontera, sus características y posible tendencia, para contribuir al desarrollo de medidas asistenciales y estructurales, teniendo en cuenta las especificidades del territorio.

\section{Concepto y clasificación de pobreza}

Es difícil establecer una única definición de pobreza, debido a los diferentes enfoques desde los cuales se puede aproximar a este concepto. No obstante, con el fin de obtener una acepción completa que responda al objetivo de esta investigación, se tomarán en cuenta tres criterios relevantes para determinar la situación de pobreza. El primero, basado en la imposibilidad de cubrir necesidades o insuficiencia de recursos, considera a la pobreza en función de la renta percibida, de forma que sea esencial obtener subsidios y ayudas sociales para alcanzar un nivel de bienestar, que posibilite satisfacer las necesidades básicas (Feres y Mancero, 2001; Núñez, 2009).

El segundo enfoque relaciona a la pobreza con el estilo de vida, entendiéndose como pobre a la persona que no pueda cumplir con los estándares sociales de vida (Townsend, 1979; Atkinson, 1987; Ruiz, 2012), perfilándose los ingresos como clave para alcanzarlos.

En tercer lugar, desde la perspectiva de las capacidades, ${ }^{3}$ Sen (1999) identifica a la pobreza con la ausencia de igualdad de capacidades de los individuos que componen la sociedad, siendo estas determinantes de su nivel de ingreso, de sus niveles de privación y, consecuentemente, de su bienestar social. Es necesaria la instrumentalización de mecanismos institucionales que ofrezcan oportunidades, para transformar las capacidades en posibles mejoras del bienestar humano (Townsend, 1970; Sen, 1999).

A partir de estos tres enfoques, se propone la definición de pobreza como aquella situación en la cual los individuos no tienen suficientes oportunidades ni capacidades que les permitan acceder a la satisfacción de sus necesidades, lo cual les confiere una situación de vulnerabilidad con serios problemas de exclusión social, debiendo recurrir, en ocasiones, a la solicitud de subsidios o ayudas asistenciales.

En cuanto a las tipologías de pobreza, entre los estudios previos llevados a cabo pueden señalarse las siguientes clasificaciones, en función del criterio analizado: pobreza objetiva y subjetiva (Feres y Mancero, 2001; Wagle,

3 Se entiende por capacidades a las habilidades que tiene un individuo para desarrollarse plenamente (Sen, 1992); algunas de ellas son fundamentales como la salud y la educación (PNUD, 2000). 
2002; Aguado y Osorio, 2015); pobreza estática o dinámica (Figueroa, 1993; Cantó y Mercader, 2001; Benach y Amable, 2004; Casanova, 2006; GarcíaLuque et al., 2009; Pérez-Mayo, 2009; Pérez-Moreno, 2009); pobreza unidimensional y multidimensional (Townsend, 1979; Mack y Lansley, 1985; Desai y Shah, 1988; Boltvinik, 1999; Atkinson, 2003; Morales, 2009; Portales, 2014); y pobreza crónica, transitoria recurrente y no recurrente (Arranz y Cantó, 2010; Cantó et al., 2012; Ayllón, 2013).

Estas tipologías no responden a la realidad existente que sobrevino a partir de la crisis actual, por ello en este trabajo se tomarán en cuenta las aportaciones realizadas por Cantó et al. (2012), en relación con los tipos de pobreza. De esta manera, se considera pobre crónico a aquellos individuos que experimentan un episodio de pobreza cuya duración abarca todo el periodo considerado: 2008-2014. Los pobres transitorios son aquellos no considerados como crónicos, pero que experimentan episodios de pobreza. Dentro de estos se distinguen entre: transitorios recurrentes y no recurrentes (PTNR) y recurrentes (PTR). Los primeros solo experimentan un episodio de pobreza siendo este inferior al periodo considerado (2008-2014), y los segundos experimentan dos o más episodios de pobreza, identificándose por tanto un periodo de salida y otro de reentrada a la misma (Cantó et al., 2012).

En la literatura, el tratamiento de la pobreza transitoria en el ámbito de la crisis internacional, y con el nuevo escenario mundial, cobra especial importancia, por ello se le dedica un epígrafe especial en este artículo.

Discusión sobre la pobreza transitoria a la luz de los debates sobre las diferentes formas de pobreza en el mundo

Tradicionalmente y siguiendo las líneas de pobreza estática, se clasificaban como pobres a todos aquellos individuos cuyas rentas estaban por debajo de un determinado umbral o línea de pobreza establecido, durante cierto periodo, por ejemplo, un año (Cantó et al., 2012). Sin embargo, los estudios de Bane y Ellwood (1986) sobre la metodología de los episodios sirvieron de guía para que, posteriormente, Foster (2007) pudiera realizar una clasificación de pobreza en pobres crónicos y transitorios. En España, los trabajos de Bárcena y Cowell (2006) y Cantó et al. (2012), entre otros, profundizan en este tema.

Desde una perspectiva dinámica, Bárcena y Cowell (2006), Ayllón (2008) y Arranz y Cantó (2010), en función del tiempo de permanencia en la pobreza, establecen distintas tipologías, destacando la de Cantó et al. (2012), que diferencia entre la pobreza crónica y la transitoria, distinguiendo dentro 
de esta última a quienes sufren distintos episodios de pobreza ${ }^{4}$ de forma recurrente, de quienes los sufren de manera no recurrente. Así, se determina la proporción de la población que experimenta pobreza crónica frente a la transitoria, además del tiempo de permanencia en esta situación.

Para identificar a las distintas tipologías de pobreza, Cantó et al. (2012) utilizan dos criterios complementarios: el primero se basa en la metodología de los componentes (Ravallion, 1988; Duncan y Rodgers, 1991; Rodgers y Rodgers, 1993; Jallan y Ravallion, 1998), y el segundo, en la metodología de los episodios (Paz, 2002; García-Luque et al., 2008; Maurizio et al., 2008; Cantó et al., 2012).

Según el primer enfoque -el de la metodología de los componentes basado en las restricciones de liquidez-, se identifica a un individuo como pobre crónico o permanente si su renta se encuentra por debajo del umbral de pobreza durante todo el periodo determinado; y pobre transitorio a quien sufre variaciones en la renta respecto a este umbral durante dicho periodo (Ravallion, 1988; Duncan y Rodgers, 1991; Rodgers y Rodgers, 1993; Jalan y Ravallion, 1998).

Este criterio se caracteriza principalmente por su sencillez, ya que permite determinar a los pobres crónicos teniendo en cuenta los ingresos considerados. Sin embargo, cabe señalar que la falta de liquidez de las familias no solo proviene de los mercados financieros, pues podrían ahorrar o endeudarse para compensar el exceso o la falta de rentas de cualquier periodo; aunque esta evidencia parece poco razonable debido a que los hogares de baja renta se enfrentan a restricciones de créditos importantes ${ }^{5}$ (Cantó et al., 2012).

4 Según Cantó et al. (2012), se entiende por episodio de pobreza a todo intervalo ininterrumpido de pobreza independientemente del número de años o periodos que abarque, una acepción diferente de la establecida por Bane y Ellwood (1986).

5 En este sentido, Jappelli (1990) afirma que los ingresos y la renta del hogar, así como la edad del sustentador principal, son factores condicionantes para obtener un crédito en Estados Unidos. En el ámbito europeo, Kepmson (1996) argumenta que los hogares formados por jóvenes con hijos pueden encontrarse con mayores dificultades para ahorrar o para tener activos financieros y, por consiguiente, para enfrentar los periodos de baja renta. En España, Azpitarte (2011) afirma que los hogares más afectados por variaciones en la renta son los formados por una sola persona (unipersonales), con edades inferiores a los 45 años, dada su escasa disposición de activos, incluyendo la vivienda principal. De esta forma, cuando experimentan periodos de baja renta de forma recurrente, es de esperar que no la puedan cubrir con activos financieros acumulados. Cutanda (2003) indica que gran parte de la población española puede estar afectada por estas restricciones. 
Por otro lado, cabe considerar, como señala Foster (2009), que este enfoque de los componentes no presta gran importancia a la duración de los fenómenos de pobreza. Por ello se recurre al segundo criterio, basado en la metodología de los episodios, asumiendo la existencia de restricciones de liquidez y determinando que una persona es pobre crónica en función del número de periodos que permanece bajo el umbral de pobreza correspondiente. Bane y Ellwood (1986) utilizan un concepto de episodio de pobreza diferente al que propusieron estos autores, definiéndolo como el intervalo temporal durante el cual un individuo está por debajo del umbral de pobreza.

Según la definición adoptada por Cantó et al. (2012), teniendo en cuenta los episodios de pobreza, se definen como pobres crónicos a quienes presentan un episodio cuya duración abarca todo el periodo determinado, mientras que su renta permanece por debajo del umbral. Respecto a los pobres transitorios, será preciso diferenciar a los que experimentan más de un episodio de pobreza, denominados recurrentes -según los estudios de Bane y Ellwood (1986) y Gardiner y Hills (1999)-, de quienes solo experimentan un único episodio continuo en un periodo, denominados no recurrentes (Cantó et al., 2012).

La OECD (2001) afirma que las personas que han salido de una situación de pobreza tienen grandes posibilidades de volver a ella a corto plazo. Con lo cual, los considerados pobres transitorios no recurrentes en algún momento tienen grandes posibilidades de experimentar episodios de pobreza de manera transitoria recurrente (Cantó et al., 2012). La diferencia de utilizar esta metodología basada en los episodios de pobreza y no otra presupone que no es posible compensar las diferencias salariales de un año con las rentas de un año distinto, como consecuencia de la existencia de restricciones de liquidez (Cantó et al., 2012).

Otra forma de identificar a los pobres crónicos es la planteada por la Unión Europea, que utiliza el indicador de Laeken para su medición, determinándose el porcentaje de individuos que son pobres en un año determinado y en los dos anteriores. Es decir, si se establece un horizonte temporal de cuatro años, se identificarán como pobres crónicos a quienes lo hayan sido, al menos, tres de cuatro, suponiendo el $75 \%$ de los últimos cuatro años analizados (Ayala, 2006; INE, 2006).

El enfoque de la metodología de los episodios, ocupado por Cantó et al. (2012), es aplicado por Torres y Guirao (2014) en sus estudios en Venezuela, donde clasifican como pobres crónicos a quienes permanecen por debajo del umbral de pobreza un determinado número de veces, en relación con los que son observados. Ayala (2008) afirma que esta clase de pobreza se caracteriza 
por ser más a largo plazo, requiriendo por tanto de políticas de mayor alcance, como las educativas o de salud.

En la pobreza transitoria se incluirían a todos aquellos individuos que estuvieron en situación de pobreza durante algún tiempo dentro del periodo considerado, ya sea que entraran en esa situación durante el transcurso de dicho periodo o que estuvieran en ella previamente al periodo objeto de estudio y salieran de ella durante este.

Para combatirla es necesario disponer de políticas de largo y corto plazo, diseñadas para atender a grupos de mayor vulnerabilidad (Carballo y Bongiorno, 2006); se requiere mejorar la estabilidad del empleo y garantizar la protección por desempleo en los periodos que así lo necesiten (Cantó et al., 2009).

\section{La pobreza en Jerez de la Frontera}

Jerez de la Frontera se ha visto gravemente perjudicado por las consecuencias de la crisis económica que han afectado de forma negativa a su tejido productivo, al empleo y a los niveles de pobreza (Arranz y Cantó, 2010; Laparra y Pérez, 2010; Colectivo Ioé, 2011; Arnal, 2013; FOESSA, 2014).

Esto se manifiesta a través de la disminución del PIBpm provincial, que experimentó un descenso de 11,7\% en el lapso 2008-2013 (INE, 2019), por la reducción de la actividad económica en el municipio -se cerraron 1.246 empresas-. Esto, a su vez, provocó elevadas tasas de paro, llegando al 40,2\% en 2013 (SIMA, 2017).

Estas graves consecuencias se han visto reflejadas también en los niveles de pobreza de la población estudiada. Aunque se carece de indicadores locales que puedan corroborarlo, existen otras variables que lo evidencian, como es el incremento de $97.2 \%$ (Cáritas, 2013) en el número de solicitudes de servicios de acogida y asistencia en la diócesis de Jerez, ${ }^{6}$ durante el periodo 2008-2012, y el aumento de 92.3\% del gasto en dotaciones económicas directas, cuyas partidas más relevantes son: alimentación, ayudas para vivienda, ropa-calzado, educación, formación, transporte y salud.

6 La diócesis de Jerez comprende de los municipios de Jerez de la Frontera, Sanlúcar de Barrameda, El Puerto de Santa María, Chipiona, Rota, Trebujena, Arcos de la Frontera, Algar, Bornos, Espera, Villamartín, Prado del Rey, El Bosque, Ubrique, Puerto Serrano, Algodonales, Zahara de la Sierra, Villaluenga del Rosario, Grazalema, El Gastor, Olvera, Alcalá del Valle, Torre Alháquime y Setenil de las Bodegas (Cáritas, 2013). 
A este incremento significativo del número de solicitudes hay que sumarle el aumento de ayudas sociales, especialmente las de carácter económico, tramitadas por las Unidades de Trabajo Social (UTS), desde el programa de atención primaria de los servicios sociales de Jerez de la Frontera en los últimos años. Estas ayudas sociales han alcanzado una tasa media de variación acumulativa para el periodo 2008-2013 de 156,3\% y para el de 2008-2014 de $163,2 \%$, resaltando el considerable incremento de las ayudas en el año 2014 (Gráfico 1). ${ }^{7}$

Otro aspecto significativo a tener en cuenta es la existencia de un comportamiento diferencial entre las distintas UTS (Gráfico 1). Por un lado, cabe señalar que La Serrana, La Granja, Las Delicias y la Zona Centro eran barrios que, tradicionalmente, no estaban afectados por la pobreza -como era el caso de San Telmo o San Benito- y que durante la crisis han experimentado un notable incremento en el número de solicitudes de ayudas económicas.

Por otro, la Barca, pedanía de Jerez de la Frontera, alcanza los niveles más bajos, por su fuerte vinculación al sector agrario y al Plan de Empleo Rural (PER). La llegada de la crisis con la destrucción del empleo ha supuesto una transformación del mapa socioeconómico del territorio objeto de estudio, sobre todo en las zonas más afectadas.

De este análisis se desprende la aparición e identificación de nuevas zonas que pueden considerarse dentro del ámbito de la pobreza en Jerez, que anteriormente eran barrios de clase media trabajadora; lo cual pone de manifiesto el surgimiento de un nuevo perfil de pobreza ad hoc proveniente de la crisis.

Ahora bien, ¿cómo se puede considerar a este segmento de población como pobre si no existe un umbral o línea de pobreza definida en el territorio? Para responder a este problema se optó por utilizar el Indicador Público de Renta de Efectos Múltiples (IPREM), como unidad de medida de esta investigación, ya que es el usado para la baremación y concesión de ayudas sociales por el Área de Bienestar Social del Ayuntamiento de Jerez, encontrándose por debajo del umbral de pobreza nacional (Tabla 1). De esta forma, se está considerando a la población con mayor incidencia en la pobreza y a la más desfavorecida por la misma.

A partir de las baremaciones realizadas con base en el IPREM, se establecen las distintas modalidades de ayudas aplicadas a los solicitantes - asistenciales, de dependencia y económicas, entre otras-. Las ayudas económicas pueden ser en metálico (cheques bancarios y nominativos) o en especie. Las metálicas están comprendidas por la económica periódica, económica puntual y el

7 El gráfico y las tablas se encuentran en el Anexo, al final del presente artículo (Nota del editor). 
salario social. ${ }^{8}$ Entre las ayudas en especie cabe mencionar la de especie en farmacia, especie periódica -la más conocida es el cheque Carrefour- y la bonificación tasa-residuos, que pueden tener carácter periódico o puntual. ${ }^{9}$

Además, paralelamente, el Área de Bienestar Social de Jerez actúa como intermediario con determinados organismos o instituciones como: Comedor Salvador, Cruz Roja y Cáritas, tramitando sus correspondientes ayudas destinadas a la alimentación y asistencia de las personas en situación de pobreza.

\section{Metodología}

A partir de la revisión de la literatura del epígrafe anterior, se observa la escasez de información necesaria para medir o cuantificar la pobreza y su perfil, para territorios a nivel local, como es el caso del municipio de Jerez. La información pública suministrada por bases oficiales como el Instituto Nacional de Estadística (INE) o el Área de Bienestar Social del Ayuntamiento de Jerez de la Frontera no es suficiente para cuantificar un concepto tan complejo. Esta constatación ha determinado la necesidad de emplear una metodología exploratoria, para obtener las variables determinantes de la situación de pobreza (tales como: ingresos de la unidad familiar, retrasos en los pagos los últimos doce meses, carencias materiales, dimensiones básicas de consumo e indicadores relacionados con los problemas de la vivienda), a través de encuestas y entrevistas.

Por una parte, se diseñó una encuesta telefónica ${ }^{10}$-siguiendo a Alaminos y Castejón (2006), Báez (2007), Díaz de Rada (2009 y 2015), Ortiz e Izquierdo (2009), Pérez (2009), Albert y Davia (2011), Tezanos y Sotomayor (2013)-, destinada a los usuarios de los servicios sociales registrados en el Área de Bienestar Social del Ayuntamiento de Jerez. Por la otra, la información obtenida de parte de los demandantes se complementa con la visión de

8 Es tramitada por la Junta de Andalucía y se puede conceder por un plazo máximo de un año. Su importe depende del nivel de ingresos de la unidad familiar y de sus componentes. 9 Información suministrada mediante una entrevista a los trabajadores sociales del Área de Bienestar Social del Ayuntamiento de Jerez de la Frontera.

10 Se optó por este tipo de encuesta, porque permite obtener información en un corto periodo, aclarar dudas al instante, realizar re-llamada si fuera necesario y tiene una mejor tasa de respuesta a bajo coste (Agudelo et al., 2010). Además, es la técnica que más se adecua a la información que se trata en esta investigación, al disponer de listados telefónicos de los usuarios objeto de estudio, los cuales son proporcionados por el Ayuntamiento con la más estricta confidencialidad. 
los oferentes de bienes, servicios y ayudas a personas en situación de precariedad, mediante entrevistas on line a los profesionales del sector vinculados directa o indirectamente con la población en esta situación (Tabla I) ${ }^{11}$

En el caso de la encuesta, se parte de una población integrada por 27.977 solicitantes de ayudas del Programa de Primera Atención en Jerez, que mediante la aplicación de un muestreo estratificado -donde los estratos se corresponden con los tipos de ayuda económica de dicho programa- se obtiene la muestra de la Tabla 2. Dadas las debilidades derivadas del proceso de la encuesta telefónica (como la localización del encuestado o la mayor facilidad de no respuesta por no estar delante el entrevistador), fue necesario entrevistar a 250 personas para obtener 103 respuestas; el nivel de confianza fue de $95,76 \%(\approx 96 \%)$, con un error muestral de $(p=q=0.5)$ y un error de precisión de $10 \%(0.1)$.

Para realizar las encuestas se tomó en cuenta el análisis de las variables categóricas; se emplearon tablas de contingencia, las cuales han permitido relacionar la pobreza del sector de la construcción con el estado civil, el género, el número de miembros que componen la unidad familiar, el nivel académico, el estatus social de los progenitores y la situación laboral.

En relación con el género, $70 \%$ de los demandantes de ayudas de primera atención en la Pobreza del Sector de la Construcción (PSC) son mujeres. Este hecho no indica que la mujer sea la sustentadora principal del hogar, ya que sigue siendo el hombre, sino que es la solicitante de ayudas, por motivos, principalmente, culturales y sociales.

En el caso de la entrevista, para seleccionar a los diferentes agentes de interés se empleó un muestreo a criterio o intencional (Martínez, 2003), con base en las principales asociaciones, fundaciones y ONG. En función del tamaño de las entidades, de su consolidación en el territorio, del impacto de su actividad en el entorno y de su relación con las instituciones territoriales, se seleccionó a las entidades que aparecen en el Anexo I, como la Asociación de la Comisión Católica Española de Migraciones (ACCEM), Cáritas diocesana, Centro de día el Salvador, Cruz Roja, Madre Coraje y Obra social San Juan Grande, entre otras, como muestra representativa de la población objeto de estudio ( $37 \%$ de la misma).

11 Todas las tablas y el gráfico se encuentran en el Anexo, al final del presente artículo (Nota del editor). 


\section{Principales resultados}

La información obtenida a partir del proceso de encuestas a los demandantes de ayudas sociales del Ayuntamiento de Jerez y de las entrevistas a los diversos agentes público-privados refleja la aparición de un segmento poblacional que responde a un "nuevo perfil de pobre", no tipificado antes de la crisis. Esta afirmación se basa en la acepción de pobreza aportada en este trabajo de investigación, atendiendo y combinando los tres enfoques de pobreza señalados con anterioridad, y se refuerza por el hecho de que el 76,5\% de los agentes entrevistados coincidieron en la aparición de este nuevo perfil de pobreza motivado por la crisis.

En este sentido, del conjunto de individuos que componen la muestra de demandantes de ayudas sociales en Jerez destaca con especial incidencia un colectivo (que representa $52,4 \%$ de la población muestreada), el cual se distingue por presentar una situación y estatus determinados antes de la crisis - por lo general, clase media-, y a partir de esta, exhibe características que permiten catalogar a este segmento poblacional como pobre, tales como:

- Encontrarse en una situación de falta de cobertura de sus necesidades básicas.

- Vivir en discordancia con los estándares y estilos de vida de su sociedad.

- No disponer o ver mermada la posibilidad de acceso a oportunidades y al desarrollo de sus capacidades.

- Necesidad de solicitar ayudas sociales.

Este segmento poblacional que no se encontraba en situación de pobreza antes de la crisis comparte, además, unas características homogéneas que definen su perfil y vuelven a poner de manifiesto la ruptura y el impacto socioeconómico de la crisis en determinadas capas o estratos poblacionales. Por tanto, se hallan en esta situación de pobreza sobrevenida por la crisis personas de entre 36 a 45 años, que estaban empleadas antes de esta -sobre todo en el sector de la construcción-, con pareja (61\% casadas y $20 \%$ constituidas como parejas de hecho), y casi en $70 \%$ de los casos con uno o dos menores a su cargo.

La gran mayoría de quienes integran dicho colectivo (72\%), con significativos periodos de cotización, en torno a 15 años, presentan bajos niveles de educación. Sin embargo, un bajo nivel educativo no se traslada a bajos salarios, sino que éstos, generalmente, están por encima de la media. También hay que señalar la fuerte presencia de la economía sumergida en el sector.

De igual forma, cabe destacar que parte de este colectivo surgido dentro del ámbito de la pobreza a partir de la crisis tiene niveles educativos inferiores 
a los de sus padres; es decir, se pone de manifiesto una cierta "involución social" a nivel educativo, aspecto determinante de la mejora social y de la capacidad de generación y aprovechamiento de oportunidades para salir de la situación de crisis.

Este colectivo proviene, mayoritariamente, de familias de clase media $(64,8 \%)$ y posee vivienda habitual en barrios de clase media-trabajadora del municipio de Jerez, que no eran considerados desfavorecidos, sino que estaban experimentando un crecimiento poblacional y económico.

En este sentido, se pone de manifiesto, a través del presente estudio, una situación de cambio o de "desplazamiento" de la pobreza entre las diferentes UTS del municipio. Mientras que antes de la recesión la pobreza se concentraba en la Zona Sur, San Benito y San Telmo, durante la misma estas zonas se han visto superadas por la creciente demanda de servicios sociales de primera atención de los barrios del Centro, La Serrana, Delicias y La Granja, que no eran tradicionalmente usuarios de dichos servicios (véase Tabla 3).

No obstante, se detecta una particular diferencia entre unas y otras: estas nuevas zonas de pobreza se caracterizan por un nuevo perfil de familias, denominado "familias normalizadas" que no presentan ningún problema de desestructuración familiar, sino que su principal problema es la falta de empleo y recursos económicos para hacer frente a necesidades básicas como alimentación, hipoteca, suministro eléctrico, entre otros. ${ }^{12}$ Estas familias normalizadas son las que se identifican en una nueva tipología de pobreza en dicho territorio, con gran presencia del sector de la construcción, como se ha visto previamente.

El 66,6\% de la muestra del sector de la construcción se encuentra en paro; esta situación provoca que sus fuentes de ingresos se concentren en las ayudas sociales, la prestación por desempleo -si esta no se les ha agotado yay la ayuda de familiares y amigos. Tan solo $7,4 \%$ tiene un empleo indefinido y declarado, mientras que $25,9 \%$ dispone de una contratación eventual o sin ningún tipo de contrato, fomentando la economía irregular de dicha zona.

En coincidencia con el recrudecimiento de la crisis, según los principales indicadores (PIB, paro, embargos y desahucios), 2013 fue el año en que un mayor número de unidades familiares componentes de la muestra entraron a formar parte de este nuevo segmento poblacional de pobreza. Dicha situación se puede justificar por el importante decremento en la actividad económica del sector de la construcción, materializado por el cierre de empresas en el municipio (13,03\% en 2010 y 16,74\% en 2009), lo cual dio lugar

12 Información suministrada por los propios trabajadores sociales del Ayuntamiento de Jerez de la Frontera. 
a un nuevo aumento de la tasa de paro y de población endeudada sin ingresos, coincidiendo con el término de las ayudas de prestación por desempleo en 2013 de las unidades familiares afectadas por la pérdida de sus empleos al inicio de la crisis.

Este nuevo tipo de pobreza responde a la cuestión de investigación planteada. La crisis ha traído consigo un cambio socioeconómico que afecta particularmente a un segmento poblacional determinado, caracterizado por amplios periodos de cotización previos a la crisis, menores niveles de formación y de oportunidades socioeconómicas que sus progenitores (generalmente de clase media), lo cual origina un serio proceso de involución social, con repercusiones a mediano y largo plazo.

Los diferentes agentes público-privados encuestados perciben que dicho colectivo tiene una tendencia a permanecer en esa pobreza, a pesar de la mejora de la situación de crisis. El 50\% opina que tendrán escasas oportunidades. En relación con las personas en situación de pobreza antes de la crisis, $61,8 \%$ considera que tendrán más dificultades para salir de la misma por sus características, fundamentalmente: baja formación, edad madura, mayor dificultad de reconversión y adaptación a las nuevas condiciones de trabajo y, sobre todo, de reinserción en el mercado laboral, además de tener que superar situaciones económicas complejas, como la pérdida de vivienda, en numerosos casos, y el alto grado de endeudamiento que muchos han contraído a largo plazo.

La escasez de información en el municipio de Jerez de la Frontera impide establecer una relación para determinar qué grupos son los más perjudicados por estas carencias y, por consiguiente, con mayor nivel de desigualdad. Dicho problema puede repercutir en las actuaciones de instituciones públicas o privadas para subsanar la cuestión. No obstante, de los datos extraídos de la encuesta se detecta que $68.93 \%$ de las unidades familiares estudiadas están compuestas por algún miembro con una edad menor a 16 años $^{13}$-padeciendo en este caso pobreza infantil-y el $24,29 \%$, por jóvenes cuyas tramos etarios se encuentran entre los 16 y 24 años. Estos dos colectivos son los más castigados por la pobreza y la desigualdad.

Cabe destacar que este tipo de pobreza lleva aparejado un incremento de la pobreza infantil, ya que casi $70 \%$ de las unidades familiares en esta situación tienen hijos menores a su cargo. Además, en España estas cifras son más elevadas que sus correspondientes medias a nivel de la Unión Europea,

13 Se utiliza la misma metodología que el INE (2018) para la clasificación de los grupos de edad, aplicándola a la variable riesgo de pobreza y exclusión social, siendo esta: menos de 16 años, de 16 a 24 años, de 25 a 49 años, de 50 a 64 años y de 65 y más años. 
agudizándose la problemática, pues la tasa de riesgo de pobreza y exclusión social es de $35.4 \%$ para menores de 16 años y de $38.7 \%$ para el colectivo de 16 a 24 años; mientras que en la UE28 es del $27.5 \%$ para el primer grupo y del $31.5 \%$ para el segundo, en el año 2014 (Eurostat, 2018). El empeoramiento de dicha situación, a partir del recrudecimiento de la crisis en España, pone de manifiesto la gravedad y la permanencia de algunos de sus impactos socioeconómicos. La circunstancia en la cual se encuentran estos menores puede estar acompañada de problemas de pobreza intergeneracionales, de forma que puede convertirse en "pobreza estructural", de la que, en la mayoría de los casos, es muy difícil salir.

Respecto a la pobreza infantil y juvenil, considerando las carencias materiales y severas, en 2015, en cuanto a los niveles de privación, el grupo de 16 a 24 años presentó el mayor porcentaje (22,7\%), seguido del de los menores de 16 años $(20,1 \%)$. En torno a la carencia material severa, el colectivo menor de 16 años alcanzó 9,1\%, mientras que el de 16 a 24 años, 8,3\%.

En cuanto al tipo de hogares con carencia material, 31,9\% estaba formado por un adulto solo con hijos dependientes, y $21,3 \%$, por una mujer sola menor de 65 años. Respecto a los hogares con carencia material severa, $12,5 \%$ estaba integrado por un adulto solo con hijos dependientes, y $11,5 \%$, por una mujer sola menor de 65 años.

Algunos indicios de esta desigualdad se manifiestan a través de las carencias materiales o niveles de privación que, según los resultados alcanzados en este estudio, son los siguientes:

En relación con los indicadores de retraso de los pagos en los últimos 12 meses, cabe señalar que $88,89 \%$ de los encuestados pertenecientes a la PSC no pueden llegar a fin de mes, $33.34 \%$ presentan retrasos en los pagos de hipoteca y alquiler, y $16.67 \%$, en pagos de compras aplazadas. Todas estas cifras ponen de manifiesto la gran problemática socioeconómica que representa la pobreza en el territorio de Jerez de la Frontera.

Los datos correspondientes a cada uno de estos indicadores muestran un ciclo evolutivo dentro de la situación de pobreza, donde la PSC se encuentra en su fase inicial, en función de las características que presenta y del año -a partir de la crisis- en el cual estas personas entraron en dicha situación.

Son individuos en una circunstancia de pobreza sobrevenida y más reciente que los otros tipos de pobreza. Esto les permite aún mantener su vivienda, aunque sea en parte gracias al apoyo familiar, pero comienzan ya a acumular retrasos en los pagos que les van, paulatinamente, dificultando la sostenibilidad de esta situación, dirigiéndolos hacia la siguiente fase del ciclo, de pobreza más arraigada y en la que se hallan los otros tipos de pobreza, donde ya se vería 
afectada incluso la posibilidad de mantener la vivienda. Se sigue poniendo de manifiesto la importancia de las ayudas de las familias como sostenimiento y apoyo de estos colectivos.

En relación con los indicadores de necesidades básicas materiales, el $100 \%$ de estos individuos no puede irse de vacaciones una vez al año, 68,5\% no tiene Internet en casa, $72 \%$ carece de lavavajillas y $31,49 \%$ no posee automóvil.

En cuanto a los indicadores de necesidades básicas de consumo, 44.5\% de los encuestados no pueden comer carne o pollo dos veces por semana -y quienes lo hacen es porque las familias se los suministran-, al $50 \%$ le supone un grave esfuerzo adquirir medicamentos sin receta médica, $62.90 \%$ no puede comprar ropa de abrigo una vez al año, $70 \%$ no puede adquirir ropa nueva cada temporada, $68,50 \%$ no puede comprar zapatos nuevos cada año y $83 \%$ no puede afrontar gastos imprevistos. Por tanto, dichas unidades familiares no poseen capacidad de ahorro. Con anterioridad se mencionó que la mayoría no llegaba a fin de mes, hecho que explica su incapacidad para afrontar gastos extraordinarios sobrevenidos. Asimismo, cabe señalar que la falta de ingresos dificulta el uso de la calefacción en los meses de frío, alcanzando la cifra del $64.81 \%$ de los hogares.

Respecto a los indicadores relacionados con los problemas de la vivienda, catalogados como de dimensión secundaria: el 99\% de las unidades familiares no puede comprar muebles cuando se les estropean, $97 \%$ no puede adquirir electrodomésticos, $26 \%$ tiene problemas de goteras y humedades, y $3 \%$, de hacinamiento. De esto se deduce que tampoco tienen liquidez suficiente para atender estas cuestiones.

$\mathrm{Al}$ relacionar este nuevo tipo de pobreza con la clasificación según la nomenclatura tradicional empleada en este trabajo (pobreza crónica, transitoria recurrente y no recurrente) (Cantó et al., 2012), se obtuvieron los siguientes datos: $5 \%$ de la PSC padece pobreza crónica, 50\%, transitoria recurrente, y $45 \%$, transitoria no recurrente, mostrando periodos de reentradas y salidas de la misma.

Este análisis permite ofrecer una visión más cercana y real de la situación de pobreza actual en el territorio de Jerez y de su carácter multidimensional. Sobre todo, para la población con dichas dificultades.

\section{Conclusiones}

Con esta investigación de carácter empírico y exploratorio se ha alcanzado el objetivo planteado en la introducción, al profundizar en el conocimiento 
de la pobreza en general y, en particular, de los factores que pueden poner de manifiesto la reciente aparición de un nuevo perfil dentro de la pobreza transitoria, a partir de la crisis económica.

Derivado del análisis de este trabajo, se ha identificado la existencia de un nuevo tipo de pobreza en el municipio de Jerez de la Frontera, sus características y su posible tendencia, lo cual puede ayudar a la mejora en el establecimiento y ejecución de actuaciones generales y específicas, con mayor vertebración de los sectores público y privado, pudiendo enriquecer el resultado de su aplicación en la población y en el territorio.

Quienes padecen este nuevo tipo de pobreza constituyen un colectivo que previamente no era considerado como "pobre", ya que no tenía falta de cobertura de sus necesidades, no estaba en discordancia con el estándar de estilo de vida y tenía la posibilidad de acceder a oportunidades y desarrollar sus capacidades. Sin embargo, su situación ha devenido en un nuevo tipo de pobreza transitoria que, según los agentes territoriales entrevistados, va a persistir cuando desaparezcan los aspectos que han provocado la crisis. Los principales factores y características que configuran el perfil específico de este nuevo colectivo son los siguientes:

Son personas de entre 36 a 45 años, con pareja (61\% son casados y $20 \%$, constituidas como parejas de hecho), casi en el $70 \%$ de los casos con uno o dos menores a su cargo - dato que refleja el incremento de la pobreza infantil del territorio-, que tienen un amplio periodo cotizado antes de la crisis, sobre todo en el sector de la construcción (sobre 15 años), con salarios más altos que la media municipal, y han contraído compromisos de pagos aplazados, pero a los cuales, al perder drásticamente su estatus, no pueden hacer frente. Se han convertido en desempleados de larga duración, con poco nivel de formación, y provienen de familias de clase media; por lo tanto, se puede hablar de una involución social.

Este nuevo grupo de pobreza vive en barrios también de clase media trabajadora no considerados desfavorecidos, pero en donde se han incrementado las solicitudes de ayudas sociales, y que hasta su entrada en la nueva situación de falta de cobertura de sus necesidades, no las requerían.

Este cambio de escenario socioeconómico, identificado y conceptualizado en el presente trabajo, requiere de actuaciones específicas, junto con medidas generales, para enfrentar las repercusiones a corto, medio y largo plazo sobre la concepción de pobreza y el bienestar de la población.

Y esta es la principal contribución del artículo: determinar la aparición de un nuevo tipo de pobreza transitoria en el municipio de Jerez de la Frontera y su perfil específico, para mejorar la aplicación de medidas público- 
privadas no solo de carácter meramente asistencial, y puedan desarrollarse actuaciones estructurales integradas, que impulsen la cohesión económica, social y territorial, teniendo en cuenta las especificidades reales y potenciales del marco donde se llevan a cabo.

Como futuras líneas de investigación, el primordial elemento por desarrollar es el diseño de la propuesta de medidas que pueden aplicarse, a partir del diagnóstico poblacional realizado y de la identificación del nuevo colectivo en situación de pobreza. Además, podrían extenderse y adaptarse estos análisis a otros territorios, de manera que se pueda ir tratando de manera rigurosa uno de los grandes problemas actuales a nivel mundial: la pobreza. Otra línea muy importante que se abre también, con base en este trabajo, es el análisis de la relación entre pobreza y desigualdad, tema prioritario para el bienestar de la población y el desarrollo territorial.

$\mathrm{Al}$ ser conscientes de las limitaciones encontradas en la búsqueda de información relacionada con la pobreza en el municipio y la falta de indicadores que permitan medir exhaustivamente la pobreza en el territorio estudiado -dado que la situación de crisis no ha cedido-, el análisis empírico de la presente investigación representa una primera aproximación o estudio inicial exploratorio. A partir de aquí queda abierto un nuevo camino para seguir trabajando en el seguimiento de la situación de pobreza durante y después de la crisis.

\section{Referencias}

Alaminos, Antonio y Castejón, Juan Luis (2006), Elaboración, análisis e interpretación de encuestas, cuestionarios y escalas de opinión, España: Universidad de Alicante, Marfil.

Albert, Cecilia y Davia, María (2011), "Pobreza monetaria, exclusión educativa, y privación material de los jóvenes", en Revista de Economía Aplicada, vol. 19, núm. 56, España: Universidad de Zaragoza.

Álvarez, Sonia (2005), "Los discursos minimistas sobre las necesidades básicas y los umbrales de ciudadanía como reproductores de la pobreza”, en Álvarez, S. [comp.], Trabajo y producción de la pobreza en Latinoamérica y el Caribe. Estructuras, discursos y actores, Argentina: CLACSO.

Aguado, Luis Fernando y Osorio, Ana. M (2015), "Percepción subjetiva de los pobres. Una alternativa a la medición de la pobreza", en Reflexión Politica, vol. 8, núm. 15, Colombia: Universidad Autónoma de Bucarawanga.

Agudelo, Gabriel et al. (2010), Metodología de las encuestas telefónicas, Colombia: Universidad de Antioquia.

Arnal, María (2013), "Crisis, desempleo y pobreza: análisis de trayectorias de vida y estrategias en el mercado laboral", en Cuadernos de Relaciones Laborales, vol. 31, núm. 2, España: Universidad Complutense de Madrid. 
Arranz, Manuel y Cantó, Olga (2010), "Measuring the effect of spell recurrence on poverty dynamics", en Wider Working Paper, núm. 072, Finlandia: United Nations University.

Atkinson, Anthony Barnes (1987), “On The Measurement of Poverty”, en Econometrica, núm. 55, vol. 4, Estados Unidos: The Econometry Society.

Atkinson, Anthony (2003), "Multidimensional Deprivation: Contrasting Social Welfare and Counting Approaches", en Journal of Economic Inequality, núm. 1, Inglaterra: London School of Economics and Political Sciencie.

Ayala, Luis (2006), "La monitorización de la desigualdad y la exclusión social: hacia un sistema integrado de indicadores", en Vidal Fernández, Fernando [dir.], $V$ Informe de FUHEM de politicas sociales: Exclusión social y estado de bienestar en España, España: Fundación de Hogar del Empleado.

Ayala, Luis (2008), "Crecimiento económico políticas públicas y bienestar: ¿el cambio de modelo?”, en Hernández, M. [coord.], Exclusión socialy desigualdad, España: Ediciones la Universidad de Murcia.

Ayllón, Sara (2008), "Modelling Poverty transitions in Spain: Do attrition and initial conditions really matter?", en UNU-WIDER Conference on "Frontiers of Poverty Analysis", Finlandia: United Nations University.

Ayllón, Sara (2013), "Understanding poverty persistence in Spain”, en SERIEs. Journal of the Spanish Economic Association, vol. 4, núm. 2, España: Asociación Española de Economía.

Azpitarte, Francisco (2011), "Measurement and Identification of Asset-Poor Households: A Cross-National Comparison of Spain and the United Kingdom", en The Journal of Economy in Inequality, vol. 9, núm. 1, Estados Unidos: Springer.

Báez, Juan (2007), Investigación cualitativa, España: Escuela Superior de Gestión Comercial y Marketing.

Bane, Mari Jo y Ellwood, David (1986), "Slipping into and out of poverty: the dynamics of spells”, en Journal of Human Resources, vol. 21, núm. 1, Estados Unidos: The University Wisconsin Press Journals Division.

Bárcena, Elena y Cowell, Frank (2006), "Static and Dynamic Poverty in Spain 1993-2000", en Hacienda Pública Española. Revista de Economía Pública, núm. 179, vol. 4, España: Instituto de Estudios Fiscales y Ministerio de Hacienda.

Benach, Joan y Amable, Marcelo (2004), "Las clases sociales y la pobreza”, en Gaceta Sanitaria, vol. 18, núm. 1, España: Sociedad Española de Salud Pública y Administración Sanitaria.

Boltvinik, Julio (1999), "Métodos de medición de la pobreza. Conceptos y tipología", en Socialis. Revista Latinoamericana de Politica Social, núm. 1, Argentina: Flacso, Universidad Nacional de Rosario y Universidad de Buenos Aires.

Cantó, Olga y Mercader, Magda (2001), "Young people leaving home: The impact of poverty in Spain”, en Bradbury, B. et al. [eds.], The Dynamics of Child Poverty in Industrialised Countries, Reino Unido: Cambrigde University Press.

Cantó, Olga et al. (2009), "La dinámica de la pobreza en España: duración, tipología y flujos", en XVI Encuentro de Economía Pública, 5 y 6 de febrero, España. Disponible en: https://dialnet.unirioja.es/servlet/libro?codigo=360583 [14 de mayo de 2016].

Cantó, Olga et al. (2012), "Pobreza crónica, transitoria y recurrente en España”, Revista de Economía Aplicada, vol. 58, núm. 20, España: Universidad de Zaragoza. 
Carballo, Jerónimo y Bongiorno, María (2006), "La evolución de la pobreza en Argentina: crónica, transitoria, diferencias regionales y determinantes (1995-2003)”, en Documento de Trabajo del CEDLAS, núm. 35. Argentina: Universidad de la Plata.

Cáritas (2013), "Empobrecimiento y desigualdad social: El aumento de la fractura social en una sociedad vulnerable que se empobrece", en VIII Informe del Observatorio de la Realidad Social, España: Cáritas Española.

Casanova, Luis (2006), "Análisis estático y dinámico de la pobreza en Argentina: Evidencia empírica para el período 1998-2002", en Documento de Trabajo, núm. 31, Argentina: Universidad Nacional de la Plata.

Colectivo Ioé (2011), "Efectos sociales de la crisis. Una evaluación a partir del Barómetro social de España”, en Papeles de relaciones ecosociales y cambio global, núm. 113, España: FUHEM Educación + Ecosocial.

Cutanda, Antonio (2003), "An empirical investigation of the effect of borrowing constraints on Spanish consumption”, en Spanish Economic Review, vol. 5, núm. 1, España: Springer.

Desai, Meghnad y Shah, Anup (1988), "An econometric approach to the measurement of poverty”, en Oxford Economic Papers, vol. 40, núm. 3, Inglaterra: Oxford University Press.

Díaz de Rada, Vidal (2009), Análisis de datos de encuestas: Desarrollo de una investigación completa utilizando SPSS, España: Universitat Oberta de Catalunya.

Díaz de Rada, Vidal (2015), Manual de trabajo de campo en la encuesta: presencial y telefónica, España: Centro de Investigaciones Sociológicas.

Duncan, Gregy Rodgers, Willard (1991), "Has children’s Poverty Become More Persistent?", en American Sociological Review, vol. 56, Estados Unidos: American Sociological Association.

EAPN [European Antipoberty Network] (2017), $7^{\circ}$ Informe. El estado de la pobreza. El seguimiento del indicador de riesgo de pobreza y exclusión social en España (2008-2016). Disponible en: http://www.eapn.es/estadodepobreza/ARCHIVO/documentos/ Informe_AROPE_2017_Resumen_Ejecutivo.pdf [10 de enero de 2018].

EAPN [European Antipoberty Network] (2016), "El estado de la pobreza en Andalucía. La pobreza invisible”. Disponible en: http://lapobrezainvisible.es/wpcontent/ uploads/2016/03/Informe_EAPNA.pdf [15 de diciembre de 2017].

Eurostat (2018), "People at risk of poverty or exclusión social by age and sex". Disponible en: https://appsso.eurostat.ec.europa.eu/nui/submitViewTableAction.do $[25 \mathrm{de}$ noviembre de 2018].

Feres, Juan Carlos y Mancero, Xavier (2001), Enfoques para la medición de la pobreza. Breve revisión de la literatura, Chile: CEPAL.

Figueroa, Adolfo (1993), "Estática y dinámica en el análisis económico”, en Economía, vol. 16, núm. 32, Perú: Pontificia Universidad Católica de Perú.

FOESSA [Fundación de Fomento de Estudios Sociales y Sociología Aplicada] (2014), VII Informe sobre exclusión social y desarrollo social en España, Madrid: Cáritas y Fundación FOESSA.

Foster, James (2007), “A Class of Chronic Poverty Measures”, en Working Paper, núm. 07W01, Estados Unidos: Department of Economics, Vanderbilt University.

Foster, James (2009), “A Class of Chronic Poverty Measures”, en Addison, T., Hulme, D. y Kanbur, R. [eds.], Poverty Dynamics Interdisciplinary Perspectives, Reino Unido: Oxford University Press. 
Convergencia Revista de Ciencias Sociales, vol. 27, 2020, Universidad Autónoma del Estado de México

García-Luque, Olga et al. (2009), "Disparidad territorial de la Pobreza Dinámica en España”, en Estudios de Economía Aplicada, vol. 27, núm. 2, España: Universidad de Zaragoza.

Gardiner, Karen y Hills, John (1999), "Policy implications of new data on income mobility", en The Economic Journal, vol. 109, núm. 453, España: Universidad de Zaragoza.

GUSS [Gestión de Usuarios de Servicios Sociales] (2016), Base de datos del Área de Bienestar Social del Ayuntamiento de Jerez de la Frontera. Disponible en: https://www.jerez.es/ webs_municipales/accion_social/recursos/area_de_servicios_sociales/ [14 de mayo de 2016].

INE [Instituto Nacional de Estadística] (2006), La pobreza y su medición. Presentación de diversos métodos de obtención de medidas de pobreza. Disponible en: https://www.ine. es/daco/daco42/sociales/pobreza.pdf [25 de noviembre de 2018].

INE [Instituto Nacional de Estadística] (2018), Salario, ingresos y exclusión social. Consulta de tasa de riesgo de pobreza y exclusión social por grupos de edad. Disponible en: https:// www.ine.es/daco/daco42/sociales/pobreza.pdf [25 de noviembre de 2018].

INE [Instituto Nacional de Estadística] (2018), "Umbral riesgo de pobreza (valores ilustrativos)". Disponible en: http://www.ine.es/jaxiT3/Datos.htm?t=9964 [25 de noviembre de 2018].

INE[Instituto NacionaldeEstadística] (2019), “ContabilidadRegionaldeEspaña.Base2010. Serie homogénea. Resultados provinciales. Serie contable 2000-2016. PIB a precios de mercado y Valor añadido bruto a precios básicos por ramas de actividad”. Disponible en: http://www.ine.es/dynt3/inebase/es/index.htm?type=pcaxis\&path=/t35/p010/ base2010/homoge \&file $=$ pcaxis $\& \mathrm{dh}=0 \&$ capsel $=2^{\wedge}[12$ de junio de 2019].

IPREM [Indicador Público de Renta y Efectos Múltiples] (2016), Disponible en: http:// www.iprem.com.es/ [13 de junio de 2016].

Jalan, Jyotsna y Ravallion, Martín (1998), “Transient Poverty in Post-reform Rural China”, en Journal of Comparative Economics, vol. 26, núm. 2, Estados Unidos: Association for Comparative Economic Studies.

Jappelli, Tullio (1990), "Who is credit constrained in the U.S. Economy?", en Quarterly Journal of Economics, vol. 105, núm. 1, Reino Unido: Oxford University Press.

Kempson, Elaine (1996), Life on a low Income, Estados Unidos: Joseph Rowntree Foundation.

Laparra, Miguel y Pérez, Begoña (2010), El primer impacto de la crisis en la cohesión social en España, España: Cáritas Española.

Mack, Joan y Lansley, Stewart (1985), Poor Britain. Poverty and Social Exclusion, Inglaterra: Allen and Urwin.

Malgesini, Graciela y Candalija, Joan (2014), Dossier Pobreza de EAPN España. Disponible en: http://www.eapn.es/ARCHIVO/documentos/dossier_pobreza.pdf [15 de diciembre de 2017].

Martínez, Valentín (2003), Diseño de encuestas de opinión, España: Ra-Ma.

Maurizio, Rosana et al. (2008), Dinámica de la pobreza y el mercado de trabajo en Argentina post-convertibilidad, Argentina: Ministerio de Trabajo, Empleo y Seguridad Social.

Morales, Eduardo (2009), "La evolución de la pobreza difusa multidimensional en México 1994-2006”, en Working Papers, núm. 2009-4, Ciudad de México: Banco de México. Disponible en: https://www.econstor.eu/bitstream/10419/83704/1/595903738.pdf [25 de noviembre de 2018]. 
Núñez, José Javier (2009), "Estado actual y nuevas aproximaciones a la medición de la pobreza”, en Estudios de Economía Aplicada, vol. 27, núm. 2, España: Universidad de Zaragoza.

OECD (2001), "When money is tight: poverty dynamics in OECD countries", en OECD Employment Outlook, Francia: Organization for Cooperation and Economic Development. Disponible en: http://www.oecd.org/els/emp/2079296.pdf [14 de mayo de 2016].

Ortiz, Salvador y Gil, María (2009), "Determinantes de la pobreza extrema en España desde una doble perspectiva: monetaria y de privación”, en Estudios de Economía Aplicada, vol. 27, núm. 2, España: Universidad de Zaragoza.

Paz, Jorge (2002), "Una introducción a la dinámica de la pobreza en la Argentina”, en Serie Documentos de Trabajo, núm. 226, Argentina: Universidad del Centro de Estudios Macroeconómicos.

Pérez, Jesús (2009), “Un análisis dinámico de la privación en España”, en Estudios de Economía Aplicada, vol. 27, núm. 2, España: Universidad de Zaragoza.

Pérez, Salvador (2009), "El estudio de la pobreza en España desde una óptica económica: medición y políticas", en Estudios de Economía Aplicada, vol. 27, núm. 2, España: Universidad de Zaragoza..

PNUD (2000), Human Development Report 2000, Estados Unidos: Oxford University Press.

Portales, Luis (2014), "Capital social y pobreza multidimensional, el caso de los portales de Monterrey, México”, en Convergencia. Revista de Ciencias Sociales, núm. 66, México: Universidad Autónoma del Estado de México. Disponible en: https://convergencia. uaemex.mx/article/view/1769 [05 de junio de 2019].

Ravallion, Martín (1988), "Expected poverty under risk-induced welfare variability”, en The Economic Journal, vol. 98, núm. 393, Reino Unido: Oxford Academic.

Rocha, Fernando (2012), "La crisis económica y sus efectos sobre el empleo en España", en Gaceta Sindical. Reflexión y debate, núm. 19, España: Confederación Sindical de Comisiones Obreras. Disponible en: https://www.ccoo.es/04f1f09eb78ccebccd4265 1e9fe8c064000001.pdf [13 de junio de 2019].

Rodgers, Joan y Rodgers, John (1993), “Chronic Proverty in the United States”, en Journal of Human Resources, núm. 18, Estados Unidos: University of Wisconsin Press.

Ruiz, Naxhelli (2012), "La definición y medición de la vulnerabilidad social. Un enfoque normativo”, en Investigaciones Geográficas, núm. 77, México: Universidad Nacional Autónoma de México.

Sánchez-López, Celia y Paz-Báñez, Manuela (2016), "Desigualdad y pobreza en la Gran Recesión. Diferencias entre los países de la UE”, en Revista de Economia Mundial, núm. 44, España: Universidad de Huelva.

Sen, Amartya (1992), Inequality Re-examined, Estados Unidos: Harvard University Press.

SIMA [Sistema de Información Multiterritorial de Andalucía] (2017), "Explotación obtenida a partir de la tasa municipal de desempleo. Media anual”. Disponible en: https://www.juntadeandalucia.es/institutodeestadisticaycartografia/iea/ consultasActividad.jsp?CodOper $=104 \&$ sub=38120 [10 de noviembre de 2017].

Tezanos, José Luis y Sotomayor, Eva María (2013), En los bordes de la pobreza: las familias vulnerables en contextos de crisis, España: Biblioteca Nueva. 
Torres, Elisabeth y Guirao, Gines (2014), “Determinantes de la pobreza dinámica según el enfoque de los episodios”, en Anales de Economía Aplicada, núm. 28, España: ASEPELT-España y Delta Publicaciones.

Townsend, Peter (1970), "Concept of poverty", en International Seminar on Poverty, Estados Unidos: American Elsevier.

Townsend, Peter (1979), Poverty in the United Kingdom, Inglaterra: Penguin Books.

Vite Pérez, Miguel Ángel (1999), "Amartya Kumar Sen: notas para pensar la pobreza y la desigualdad social”, en Sociológica, año 14, núm. 39, México: Universidad Autónoma Metropolitana.

Wagle, Udaya (2002), "Volver a pensar la pobreza: definición y mediciones", en Revista Internacional de Ciencias Sociales, núm. 171, vol. 54. Disponible en: https://www.oei. es/historico/salactsi/rics171.htm [13 de junio de 2019].

\section{Anexo}

Entidades que colaboran con la pobreza en Jerez de la Frontera

Consumo

-Asociación familiares y amigos centro español de solidaridad jerezana (Afacesje), Asociación Brote de vida, Centro de Acogida la Salle, Fundación Hogar san Juan, Monasterio de Belén-La Cartuja.

Conventos: las Mínimas, José Barja clarisas, Hermanas reparadoras, Madre de Dios, Santa María de la GraciaAgustinas.

Reparto

Asamblea cristiana de Jerez,

Asociaciones: Afectados por la hipoteca, Colectivo Tio Juane, Cristiana evangélica la Granja, Chiquitín (de mayores), Manos abiertas (de mujeres), De mujeres igualdad y progreso. Asociaciones de vecinos: El mirador, el Portal, San Juan de Dios.

Centro de Acogida de Inmigrantes (Ceain).

Consumo de alimentos Asociaciones: Esperida, Voluntariado por otro mundo.

Reparto de alimentos Asociaciones: Voluntariado libre, Benéfica obra social vida y esperanza, El Samaritano, Iniciativa Solidaria Jerezana, Red Madre Coraje, Tharsis Bethel, Vida Abundante, Voluntariado Libre.

Obra social Hospital San Juan Grande, Iglesia cristiana manos de alfarero, Iglesia Puerta del cielo.

Asociación de vecinos: $\mathrm{M}^{\mathrm{a}}$ Auxiliadora, San Jerónimo y Barriada las flores. 
Consumo + Reparto de Convento Hermanas de la cruz.

alimentos

Asociaciones: Me importas Jerez, Flores, Solidaridad Jerezana

(Soje), Evangélica Vida Abundante, La Gran Comisión, Voluntarios por otro mundo, Esperida, Tío Juane, Brote de

Vida

Fundación escuelas y cocinas El Salvador, Comisión católica. Cáritas parroquiales: Santiago, San Mateo, Andrés y los Albarizones, San Rafael y San Gabriel, Nuestra Señora del Pilar, San Pedro, San Juan de Ávila, Santa Ana, San Miguel Arcángel, El Salvador y San Dionisio, El Perpetuo Socorro, Los 4 evangelistas, San Juan Bosco de los Descalzos, Las viñas Escuelas y cocinas el Salvador, de San Enrique y Santa Teresa de Guadalcín, Corpus Christi, La Inmaculada-Torresoto, San Juan Grande y la Calendaria, Sta Ma Madre de la Iglesia de la Granja, San Juan Grande y la Candelaria, San Benito, Madre de Dios y Cocina Autogestiona.

Centros, comedores sociales e instituciones que ayudan a personas en situación de pobreza
Asociación de la Comisión Católica Española de Migraciones (Accem), Comedor El Salvador, Centro de día el Salvador, De rehabilitación y reinserción Adonais, Centro de Acogida de Inmigrantes (Cenain), Cruz roja, Siloe, Caritas Diocesana, Salud Mental comunitaria, Unidad Hospitalaria, Distrito Sanitario, Hogar la Salle, Centro de Acogida San José.

Centros sociales que ayudan a personas en San Telmo, Montealegre, Madre de Dios, Jerez Centro, La Serrana, La Granja, San Benito, Las Delicias.

situación de pobreza en

Jerez

Trabajadores sociales de las distintas UTS que UTS: 1. Serrama, 2. San Benito, 3. La Granja, 4. Las ayudan a personas en Delicias, 5. Sur, 6. Centro, 8. San Telmo situación de pobreza en Jerez

Fuente: Elaboración propia. 


\section{Gráfico 1}

Evolución de las ayudas económicas por UTS

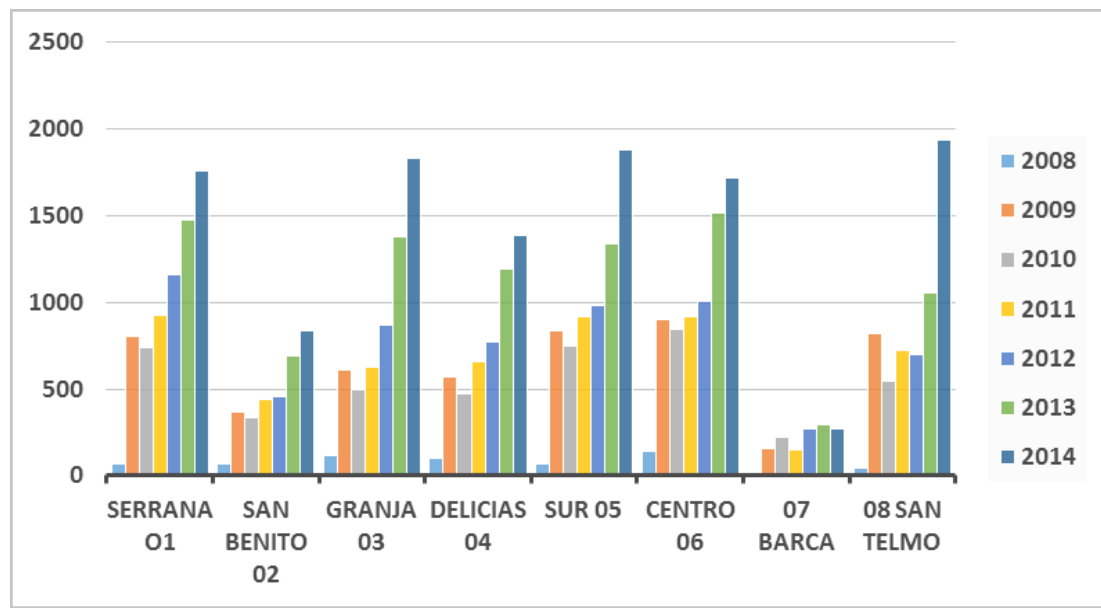

Fuente: Elaboración propia a partir de Gestión de Usuarios de Servicios Sociales (GUSS, 2016) del Ayuntamiento de Jerez de la Frontera. 


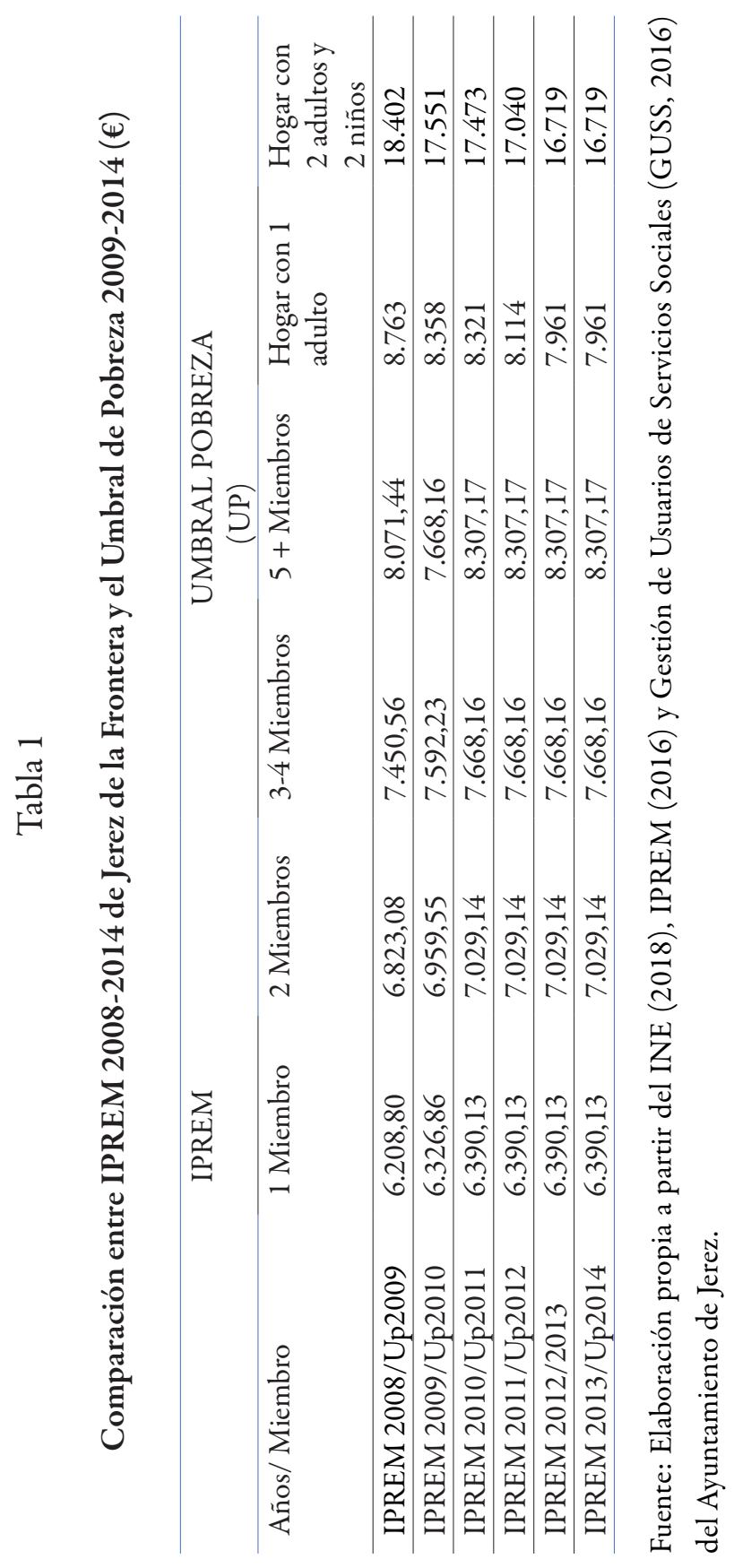




\section{Tabla 2}

Detalle de la muestra 2008-2014

\begin{tabular}{lccc}
\hline \multicolumn{1}{c}{ Estratos } & $\begin{array}{c}\text { Ayudas totales } \\
\text { por tipo }\end{array}$ & $\begin{array}{c}\text { Núm. de personas } \\
\text { solicitantes de ayuda }\end{array}$ & $\begin{array}{c}\text { MAS sin } \\
\text { reemplazamiento }\end{array}$ \\
\hline En especie farmacia & 322 & 258 & 1 \\
\hline Comedor Salvador & 512 & 453 & 2 \\
\hline Solicitud Cáritas & 845 & 796 & 3 \\
\hline Emergencia social & 957 & 468 & 2 \\
\hline Cruz Roja alimentación & 2.563 & 2.494 & 9 \\
\hline En especie puntual & 3.002 & 1.987 & 7 \\
\hline Económica puntual & 4.770 & 2.237 & 8 \\
\hline En especie periódica & 4.542 & 3.827 & 14 \\
\hline Bonificación tasa residuos & 5.314 & 2.592 & 10 \\
\hline Económica periódica & 7.957 & 4.900 & 18 \\
\hline Salario social & 11.477 & 7.965 & 29 \\
\hline Total & 42.261 & 27.977 & 103 \\
\hline
\end{tabular}

Fuente: Elaboración propia.

"Muestreo Aleatorio Simple.

Tabla 3

\section{Zonas afectadas por la pobreza del sector de la construcción}

\begin{tabular}{cc}
\hline UTS/Sector & Construcción \\
\hline Centro & $22.22 \%$ \\
\hline Serrana & $20.37 \%$ \\
\hline Las Delicias & $14.56 \%$ \\
\hline La Granja & $14.56 \%$ \\
\hline Sur & $9.80 \%$ \\
\hline San Telmo & $9.25 \%$ \\
\hline San Benito & $5.50 \%$ \\
\hline La Barca & $3.74 \%$ \\
\hline Total & $100 \%$
\end{tabular}

Fuente: Elaboración propia. 
Ma del Carmen Pérez-González. Profesora Titular del Área de Economía Aplicada del Departamento de Economía General de la Universidad de Cádiz; Coordinadora del Máster Oficial Interuniversitario en Economía y Desarrollo Territorial; Responsable del Grupo de Investigación SEJ-313 "Globalización y Dinámica Territorial". Principales líneas de investigación: economía espacial, desarrollo regional y local, economía social, Unión Europea, movimientos migratorios, nuevas tendencias de desplazamientos de población, economía del turismo. Publicaciones recientes: Pérez, M. C. y Valiente Palma, L. (2017), "La localización sectorial del cooperativismo: una aproximación a nivel territorial español”, en Revesco. Revista de Estudios Cooperativos, núm. 123, España: Universidad Complutense de Madrid; Pérez, M. C. y Lutsak-Yaroslava, N. V. (2017), "La producción científica sobre la innovación social para el desarrollo local. Una revisión bibliométrica”, en Prisma Social, núm. 19, España: Fundación iS+D para la Investigación Social Avanzada; Pérez, M. C. y Maza, F. (2018), "Determinants of education quality in the Canal del Dique y Zona Costera Region of Colombia”, en Journal of International Studies, núm. 11, Polonia, Rumanía, República Checa: University of Szczecin (Poland), Academy of Economic Studies in Bucharest (Romania) y University College of Business in Prague (Czech Republic).

Mercedes Jiménez-García. Profesora Contratada Doctora en la Universidad de Cádiz (Facultad de Ciencias Sociales y de la Comunicación, Departamento de Economía General). Subdirectora del Instituto Universitario de Investigación para el Desarrollo Social y Sostenible (INDESS) (UCA). Principales líneas de investigación: economía del turismo, desarrollo sostenible y agroalimentación. Publicaciones recientes: Jiménez García, M., Peña Sánchez, A. R. y Ruiz Chico, J. (2016), "Bipolaridad turística en Europa. La consolidación de destinos maduros en época de crisis", en Cuadernos de Turismo, vol. 38, España: Universidad de Murcia; Jiménez García, M., Ruiz Chico, J. y Peña Sánchez, A. R. (2017), "La demanda turística internacional: recuperación de la crisis y turismo de lujo, una primera aproximación al caso español”, en Revista de Investigaciones Regionales, vol. 38, España: Asociación Española de Ciencia Regional; Ruiz Chico, J., Peña Sánchez, A. R. y Jiménez García, M. (2018), "Análisis comparativo del diferencial de precios agrarios entre productores y consumidores en Europa, bajo criterios de seguridad alimentaria de abastecimiento", en Revista Cuadernos de Economía, vol. 42, núm. 12, España: Asociación Cuadernos de Economía. 
María del Carmen Pérez-Peña. Doctora por la Universidad de Cádiz. Profesora sustituta interina en la Universidad de Cádiz del Departamento de Economía General. Investigadora del Instituto Universitario de Investigación para el Desarrollo Social y Sostenible INDESS (UCA). Principales líneas de investigación: pobreza, sus tipologías y la crisis económica en España, y desarrollo local, desde: la perspectiva de la ruralidad del territorio y la del transporte transfronterizo marítimo. Publicaciones recientes: PérezPeña, M. (2017), "La crisis económica: efectos sobre las distintas tipologías de pobreza a nivel local en España", en Panorama Económico Journal, vol. 25, núm. 3, Colombia. Universidad de Cartagena; Pérez- Peña, M. (coord.) (2019), Análisis socioeconómico de las entidades locales autónomas y barriadas rurales de Jerez de la Frontera, España: Dykinson; Pérez-Peña, M. (2016), "Una aproximación exploratoria a las tipologías de pobreza en Jerez de la Frontera”, en Integraciones y desintegraciones sociales, pobreza, migraciones y refugios, España: Asociación Castellano Manchega de Sociología. 